\title{
Insectos asociados al agroecosistema de café bajo sombra en Milpuc, Amazonas, Perú
}

\author{
Angel Fernando Huaman Pilco ${ }^{1}$ D, Santos Triunfo Leiva Espinoza ${ }^{1}$, Segundo Manuel Oliva Cruz ${ }^{1}$ \& \& \\ Manuel Alberto Hernández May ${ }^{2}$ (D)
}

1. Universidad Nacional Toribio Rodríguez de Mendoza de Amazonas (UNTRM-A). Instituto de Investigación para el Desarrollo Sustentable de Ceja de Selva, Perú; anfer.hp13@gmail.com, santos.leiva@untrm.edu.pe, soliva@indesces.edu.pe

2. Universidad Juárez Autónoma de Tabasco, División Académica de Ciencias Biológicas, Código Postal 86150, Carretera Villahermosa-Cárdenas km $0.5 \mathrm{~s} / \mathrm{n}$ entronque a Bosques de Saloya, Villahermosa, Tabasco, México; manuel.hz.may@gmail.com

Recibido 23-VII-2020 • Corregido 7-X-2020 • Aceptado 19-X-2020

DOI: https://doi.org/10.22458/urj.v12i2.3144

\begin{abstract}
Insects associated with a shaded coffee agroecosystem in Milpuc, Amazonas, Peru". Introduction: Coffee agroecosystems serve as refuge to some species, favoring biodiversity, but studies in the Amazon region are scarce. Objective: To quantify insects in three vertical levels in coffee plants under shade. Methods: From June to October 2018 we collected insects in the low, medium and high strata of ten coffee plants, every 15 days, with aerial insect net, aspirator and pitfall traps. Results: 1923 specimens belonging to eight orders, 35 families and 40 genera were collected. Hemiptera had the most families (9) and genera (13). Coleoptera were most abundance ( $28 \%$ of specimens). The dominant genera were Apis and Canthon, both with 108 individuals. The highest richness, abundance and diversity were recorded in the middle stratum, with 35 species, 756 individuals and with total species diversity index ${ }^{1} D=28,5$, and abundant species diversity index ${ }^{2} D=25,56$. The maximum abundance of insects coincides with high humidity and precipitation, and low temperature. The accumulation curve stabilized from sample five. Conclusion: This particular Amazon agroecosystem harbors a considerable biodiversity of insects.
\end{abstract}

Keywords: Amazonas, Coffee, Diversity, Insects, Fluctuation, Abundance.
RESUMEN. Introducción: Los agroecosistemas cafetaleros sirven de refugio a algunas especies, favoreciendo la biodiversidad, pero los estudios en la región amazónica son escasos. Objetivo: Cuantificar insectos en tres niveles verticales en cafetos bajo sombra. Métodos: De junio a octubre de 2018 recolectamos insectos en los estratos bajo, medio y alto de diez plantas de café, cada 15 días, con red de mano, aspirador y trampas de caída. Resultados: Se recolectaron 1923 ejemplares pertenecientes a ocho órdenes, 35 familias y 40 géneros. Hemiptera tuvo la mayor cantidad de familias (9) y géneros (13). Los coleópteros fueron los más abundantes ( $28 \%$ de los especímenes). Los géneros dominantes fueron Apis y Canthon, ambos con 108 individuos. La mayor riqueza, abundancia y diversidad se registró en el estrato medio, con 35 especies, 756 individuos y con índice de diversidad de especies total ${ }^{1} \mathrm{D}=28,5$, e índice de diversidad de especies abundantes ${ }^{2} D=25,56$. La máxima abundancia de insectos coincide con alta humedad y precipitación, y baja temperatura. La curva de acumulación se estabilizó a partir del quinto muestreo. Conclusión: Este agroecosistema amazónico en particular alberga una considerable biodiversidad de insectos.

Palabras clave: Amazonas, Café, Diversidad, Insectos, Fluctuación, Abundancia. 
Los agroecosistemas de Coffea arabica (Linneo, 1753) tradicional son muy importantes para el refugio de la biodiversidad (Perfecto, Rice, Greenberg, \& Van der Voort, 1996; Richter, Klein, Tscharntke, \& Tylianakis, 2007). Estos se cultivan en los trópicos del mundo, donde los insectos presentan mayor diversidad y abundancia, llegando a constituir hasta el $90 \%$ de las especies en esta zona (Young, 1982; Pimentel et al., 1992). En los cafetales los insectos constituyen un componente importante, la mayoría interactúan como controladores biológicos (parasitoides y depredadores), polinizadores, descomponedores, transmisores de enfermedades; además forman parte de la base de la cadena alimenticia de muchos vertebrados (anfibios, aves, mamíferos y reptiles) y otros artrópodos (Mera, Gallego, \& Armbrecht, 2010; Valarezo, Cañarte, \& Navarrete, 2012; Sanderson, Svensson, Van den Brink, Gunnarsson, \& Tedengren, 2018).

En el Distrito de Milpuc, los agroecosistemas de café por lo general se encuentran en el sistema de producción tradicional, bajo sombra y se consideran como no dañino para el ambiente (Ruelas, Nava, Cervantes, \& Barradas, 2014), dado que ayudan a conservar la flora y fauna. Este sistema de producción ha sido abordado en América, ya que proporciona elementos sustentables y servicios ambientales, como la captura de carbono, protección del suelo, recarga de los mantos acuíferos y tienen un valor paisajístico (Moguel \& Toledo, 2004; Anta, 2006). Por otro lado, se han realizado estudios sobre las plagas que albergan y causan pérdidas económicas para el agricultor (Waller, Bigger, \& Hillocks, 2007; Barrera, 2008; Ribeyre \& Avelino, 2012; Sánchez, 2018).

A pesar de la importancia de estos agroecosistemas de café en el mantenimiento y resguardo de la biodiversidad, no se encuentran estudios taxonómicos, biológicos y ecológicos sobre la entomofauna asociada en Milpuc. Bajo esta expectativa se planteó el presente trabajo de investigación con el objetivo de proporcionar información sobre la diversidad y fluctuación poblacional de los insectos asociados al agroecosistema de café bajo sombra en el Distrito de Milpuc, Amazonas en Perú, mediante un estudio faunístico.

\section{MATERIALES Y MÉTODOS}

Sitio de estudio: El estudio se realizó durante los meses de junio a octubre del 2018 en un agroecosistema de $C$. arabica bajo sombra de Erytrina sp. (Moté), ubicado en el Distrito de Milpuc,

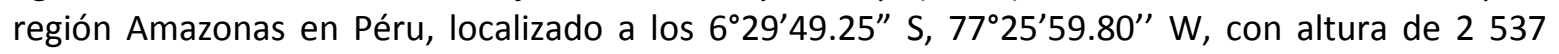
m.s.n.m. Posee un clima cálido húmedo, con temperaturas mínimas que alcanzan los $12^{\circ} \mathrm{C}$ y máximas de $30^{\circ} \mathrm{C}$. La precipitación promedio anual es de $3000 \mathrm{~mm}$ (Rojas-Briceño, Barboza, Maicelo, Oliva, \& Salas, 2019).

Se seleccionaron diez plantas de $C$. arabica al azar en media hectárea del agroecosistema de café, a cada planta seleccionada se dividió en tres estratos (bajo, medio y alto), donde el estrato bajo $0 \mathrm{~m}(\mathrm{~EB})$ representa al suelo, el estrato medio 0 a $0,40 \mathrm{~m}(\mathrm{EM})$ corresponde al tallo y el estrato alto de 0,40 a 1,50m (EA) a la copa de la planta.

Recolecta de insectos: La recolecta de insectos se realizaron cada 15 días, para los insectos presentes en el EA y EM, se utilizó una red de golpeo $(40 \mathrm{~cm}$ de diámetro por $1,20 \mathrm{~m}$ de mango) y un aspirador (\#1135A) durante 30 minutos, los insectos recolectados se depositaron en un cámara letal de Cianuro de potasio (KCN), posteriormente, se colocaron en frascos de plásticos con alcohol etílico al 70\% para su conservación (Márquez, 2005; Pérez, Hernández-May, De la Cruz, \& Sánchez, 2016).

Para recolectar a los insectos en el EB, se colocaron tres trampas de caídas Pitfall $(15 \mathrm{~cm} \mathrm{x}$ $10 \mathrm{~cm}$ ), a 30 centímetros alrededor de la planta, estas fueron cebadas con hojas, semillas y 10 frutos maduros de café, con el fin de atraer a los insectos. A cada trampa se le adicionó alcohol etílico al 70\% para la preservación de los organismos (Márquez, 2005). 
Identificación taxonómica: Los insectos recolectados se determinaron en el laboratorio de investigación de sanidad vegetal (LABISANV) de la Universidad Nacional Toribio Rodríguez de Mendoza de Amazonas (UNTRM-A), utilizando claves taxonómicas de Camacho y Quirós, (1995); Arnett y Thomas, (2000); Arnett, Thomas, Skelley, y Howard, (2002); Fernández, (2003); Triplehorn y Johnson, (2005); Trjapitzin, Myartseva, Ruíz, y Coronado, (2008); Foottit y Adler, (2009); Resh y Cardé, (2009); Rengífo-Correa y Gonzáles, (2011). La organización taxonómica se realizó de acuerdo con: Cheli, (1999) y McGavin, (2000).

Análisis de datos: Para comparar la diversidad de la entomofauna recolectada por estrato se utilizó el programa PAST: Paleontological Statistics Software Package ( $\varnothing$ yvind, Harper, \& Ryan, 2001), donde se calculó el Índice de diversidad verdadera de orden 1 ( $\left.{ }^{1} D\right)$, en donde todas las especies son consideradas en el valor de diversidad, ponderadas de manera proporcional según su abundancia en la comunidad; la medida de diversidad de orden $2\left({ }^{2} \mathrm{D}\right)$, donde se consideran las especies más comunes (Jost, 2006; 2007; Tuomisto, 2010; 2011; Moreno, Barragán, Pineda, \& Pavón, 2011). La variación de la abundancia de los insectos se comparó de manera gráfica con los valores promedios de humedad relativa (\%), precipitación $(\mathrm{mm})$ y temperatura $\left({ }^{\circ} \mathrm{C}\right)$, los cuales fueron proporcionados por la Estación meteorológica Huambo, del Instituto de Investigación para el Desarrollo Sustentable de Ceja de Selva-INDES-CES.

Se evaluó con el programa EstimateS 9, la eficiencia del muestreo mediante curvas de acumulación de especies, donde se aleatorizó 1000 veces la posición de cada unidad de esfuerzo muestreal con los estimadores no paramétricos de Chao 1 y Chao 2 (Moreno, 2001; Colwell, 2013). Esta fue juzgada gráficamente en términos de su comportamiento asintótico (Colwell, 2013). Estos estimadores requieren datos de presencia o ausencia, tienen más exactitud y menos sesgo cuando se trabaja con pequeñas muestras (Hortal, Borges, \& Gaspar, 2006; Willie, Petre, Tagg, \& Lens, 2012).

\section{RESULTADOS}

Se recolectaron 1923 insectos pertenecientes a ocho órdenes, 35 familias y 40 géneros. El orden Hemiptera registró el mayor número de familias con nueve, seguido por Coleoptera, Diptera e Hymenoptera con seis respectivamente. El orden Hemiptera registró mayor riqueza de géneros con 13, seguido por Coleoptera con siete. Cicadellidae y Membracidae obtuvieron el mayor número de géneros con tres cada una. Canthon y Apis con 108 individuos fueron los géneros más abundantes (Cuadro 1).

Del total de insectos recolectados, Coleoptera registró la mayor abundancia con 538 individuos, que representa el 27,98\%, seguido por Hemiptera con 522 individuos $(27,15 \%)$ e Hymenoptera con 292 (15,18\%). Neuroptera con 11 individuos (0,57\%) y Dermaptera con nueve $(0,47 \%)$ obtuvieron la menor abundancia de insectos (Fig. 1).

En el estrato medio (EM), se registró la mayor riqueza y abundancia de insectos con 35 especies y 756 especímenes, seguidos por los estratos alto (EA) con 34 y 648 y por último el estrato bajo (EB) con 31 y 519, respectivamente. Analizando la presencia de los géneros por estrato, 25 de ellos $(62,5 \%)$ se registraron en los tres estratos, diez $(25 \%)$ en dos estratos y cinco $(12,5 \%)$ en uno. De acuerdo a los índices de diversidad verdadera $\left({ }^{1} D\right.$ y $\left.{ }^{2} D\right)$, la máxima diversidad se obtuvo en el EM con ${ }^{1} D=28,53$ y ${ }^{2} D=25,56$, seguido por el EA con ${ }^{1} D=26,35$ y ${ }^{2} D=22,71$. El EB con ${ }^{1} D=12,72$ y ${ }^{2} D=$ 7,93 registró la menor diversidad (Cuadro 2). La máxima abundancia de insectos coincide con los datos de alta precipitación y humedad, y con temperatura baja (Fig. 2). 


\section{CUADRO 1}

Riqueza y abundancia de insectos recolectados en el cultivo de C. arabica bajo sombra en Milpuc, Amazonas en Perú

\begin{tabular}{|c|c|c|c|c|}
\hline Orden & Familia & Subfamilia & Género & Total \\
\hline \multirow[t]{7}{*}{ Coleoptera } & Chrysomelidae & Galerucinae & Psylliodes & 97 \\
\hline & & & Diabrotica & 83 \\
\hline & Scarabaeidae & Scarabaeinae & Canthon & 108 \\
\hline & Staphylinidae & Paederinae & Paederus & 99 \\
\hline & Coccinellidae & Coccinellinae & Sospita & 55 \\
\hline & Lycidae & Lycinae & Eurrhacus & 54 \\
\hline & Curculionidae & Entiminae & Microtrypes & 42 \\
\hline Dermaptera & Forficulidae & Forficulinae & Forficula & 9 \\
\hline \multirow[t]{6}{*}{ Diptera } & Dolichopodidae & Sciapodinae & Heteropsilopus & 71 \\
\hline & Tephritidae & Dacinae & Ceratitis & 56 \\
\hline & Xylomyidae & Xylomyinae & Solva & 48 \\
\hline & Fanniidae & Fanniinae & Fannia & 40 \\
\hline & Syrhidae & Syrphinae & Sphaerophoria & 39 \\
\hline & Drosophilidae & Drosophilinae & Drosophila & 28 \\
\hline \multirow[t]{13}{*}{ Hemiptera } & Cicadellidae & Cicadellinae & Erythrogonia & 73 \\
\hline & & & Borogonalia & 56 \\
\hline & & & Tortigonalia & 56 \\
\hline & Membracidae & Darninae & Cyphonia & 34 \\
\hline & & Stegaspinae & Licoderes & 29 \\
\hline & & Smiliinae & Eufairmairia & 16 \\
\hline & Clastopteridae & Clastopterinae & Clastoptera & 66 \\
\hline & Flatidae & Flatinae & Ormenaria & 65 \\
\hline & Aetalionidae & Darninae & Peltosticta & 45 \\
\hline & Miridae & Bryocorinae & Pycnoderes & 36 \\
\hline & Gelastocoridae & Nerthrinae & Nerthra & 31 \\
\hline & Pentatomidae & Asopinae & Podisus & 13 \\
\hline & Berytidae & Metacanthinae & Metacanthus & 2 \\
\hline \multirow[t]{6}{*}{ Hymenoptera } & Apidae & Apinae & Apis & 108 \\
\hline & Formicidae & Ponerinae & Pachycondyla & 96 \\
\hline & Vespidae & Polistinae & Polistes & 32 \\
\hline & Ichneumonidae & Mesochorinae & Mesochorus & 27 \\
\hline & Braconidae & Rogadinae & Aleiodes & 22 \\
\hline & Eulophidae & Eulophinae & Euplectrus & 7 \\
\hline \multirow[t]{2}{*}{ Lepidoptera } & Nymphalidae & Danainae & Danaus & 30 \\
\hline & Hesperiidae & Eudaminae & Urbanus & 15 \\
\hline Neuroptera & Chrysopidae & Chrysopinae & Chrysoperla & 11 \\
\hline \multirow[t]{6}{*}{ Orthoptera } & Gryllidae & Gryllinae & Gryllus & 84 \\
\hline & Tettigoniidae & Phaneropterinae & Microcentrum & 64 \\
\hline & Blaberidae & Blaberinae & Blaberus & 42 \\
\hline & Gryllotalpidae & Scapteriscinae & Scaptericus & 34 \\
\hline & & Riqueza & & 40 \\
\hline & & Abundancia & & 1923 \\
\hline
\end{tabular}




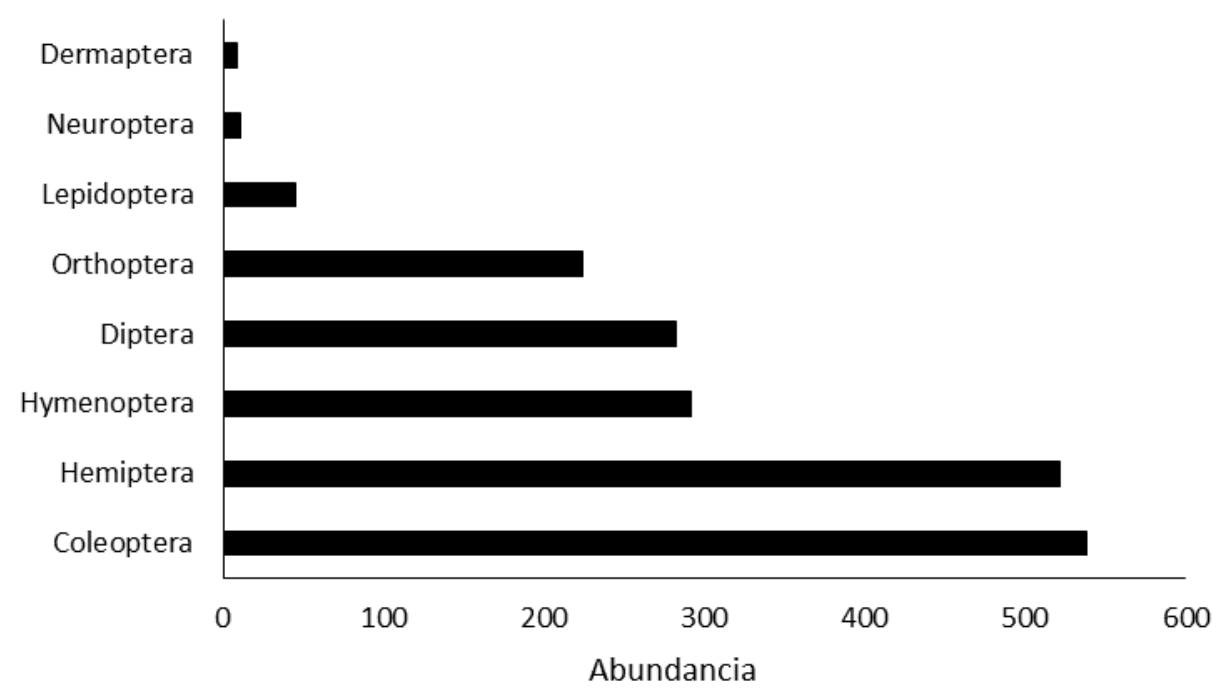

Fig. 1. Abundancia de órdenes (Insecta) en el cultivo del C. arabica bajo sombra en Milpuc, Amazonas en Perú.

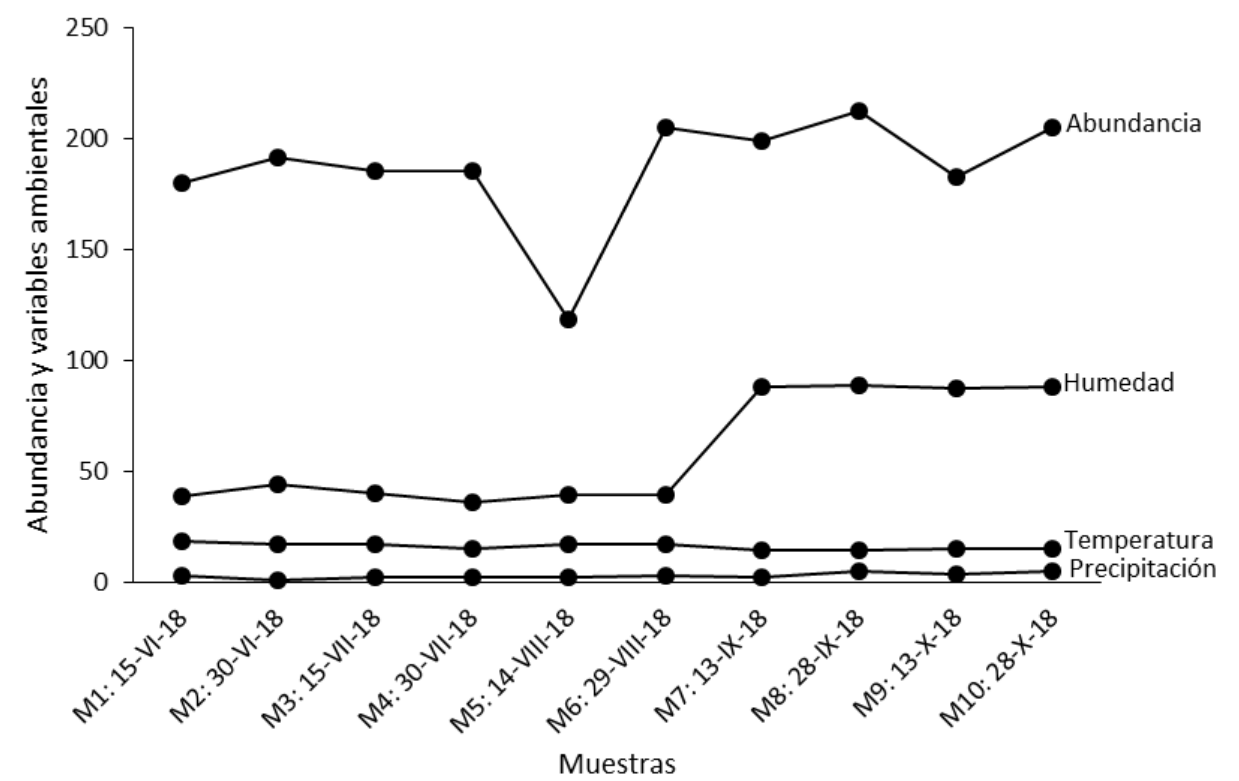

Fig. 2. Fluctuación de la abundancia de insectos y promedios quincenales de humedad, precipitación y temperatura registrados durante el periodo de muestreo en el cultivo de $C$. arabica.

La curva de acumulación de géneros observados se estabilizó a partir del muestreo cinco, lo que indica que se registró la mayoría de los insectos presentes en el cultivo de café, sin embargo, las muestras no cubren un ciclo anual y por ende no todas las estaciones climáticas, lo que podría generar una variación en la representatividad de los insectos asociados a este agroecosistema (Fig. 3). 


\section{CUADRO 2}

Abundancia de insectos recolectados por estrato en el cultivo de $C$. arabica bajo sombra en Milpuc, Amazonas en Perú

\begin{tabular}{|c|c|c|c|c|}
\hline \multirow[b]{2}{*}{ Géneros } & \multicolumn{3}{|c|}{ Estratos } & \multirow{2}{*}{$\begin{array}{l}\text { Intersección } \\
\text { estratos }\end{array}$} \\
\hline & EB & EM & EA & \\
\hline Aleiodes & 4 & 10 & 8 & $a$ \\
\hline Apis & 10 & 50 & 48 & a \\
\hline Blaberus & 11 & 29 & 2 & a \\
\hline Borogonalia & 4 & 20 & 32 & a \\
\hline Canthon & 108 & 0 & 0 & c \\
\hline Ceratitis & 4 & 41 & 11 & a \\
\hline Chrysoperla & 0 & 5 & 6 & $b$ \\
\hline Clastoptera & 3 & 20 & 43 & a \\
\hline Cyphonia & 10 & 12 & 12 & a \\
\hline Danaus & 6 & 16 & 8 & a \\
\hline Diabrotica & 9 & 44 & 30 & a \\
\hline Drosophila & 2 & 18 & 8 & a \\
\hline Erythrogonia & 5 & 29 & 39 & a \\
\hline Eufairmairia & 4 & 6 & 6 & a \\
\hline Euplectrus & 0 & 2 & 5 & $b$ \\
\hline Eurrhacus & 2 & 28 & 24 & a \\
\hline Fannia & 0 & 28 & 12 & $b$ \\
\hline Forficula & 9 & 0 & 0 & c \\
\hline Gryllus & 6 & 40 & 38 & a \\
\hline Heteropsilopus & 1 & 43 & 27 & a \\
\hline Licoderes & 2 & 12 & 15 & a \\
\hline Mesochorus & 0 & 15 & 12 & $b$ \\
\hline Metacanthus & 0 & 1 & 1 & $b$ \\
\hline Microcentrum & 2 & 36 & 26 & a \\
\hline Microtrypes & 11 & 23 & 8 & a \\
\hline Nerthra & 31 & 0 & 0 & c \\
\hline Ormenaria & 0 & 27 & 38 & $b$ \\
\hline Pachycondyla & 96 & 0 & 0 & c \\
\hline Paederus & 98 & 1 & 0 & b \\
\hline Peltosticta & 2 & 18 & 25 & a \\
\hline Podisus & 3 & 6 & 4 & a \\
\hline Polistes & 5 & 16 & 11 & a \\
\hline Psylliodes & 14 & 42 & 41 & a \\
\hline Pycnoderes & 0 & 18 & 18 & $b$ \\
\hline Scaptericus & 34 & 0 & 0 & c \\
\hline Solva & 0 & 28 & 20 & $b$ \\
\hline Sospita & 18 & 21 & 16 & a \\
\hline Sphaerophoria & 0 & 24 & 15 & $b$ \\
\hline Tortigonalia & 2 & 19 & 35 & a \\
\hline Urbanus & 3 & 8 & 4 & $\mathrm{a}$ \\
\hline Riqueza & 31 & 35 & 34 & \\
\hline Abundancia & 519 & 756 & 648 & \\
\hline${ }^{1} \mathrm{D}$ & 12,72 & 28,53 & 26,35 & \\
\hline${ }^{2} \mathrm{D}$ & 7,93 & 25,56 & 22,71 & \\
\hline
\end{tabular}

Géneros registrados: a en tres estratos, b en dos estratos, c en un estrato 


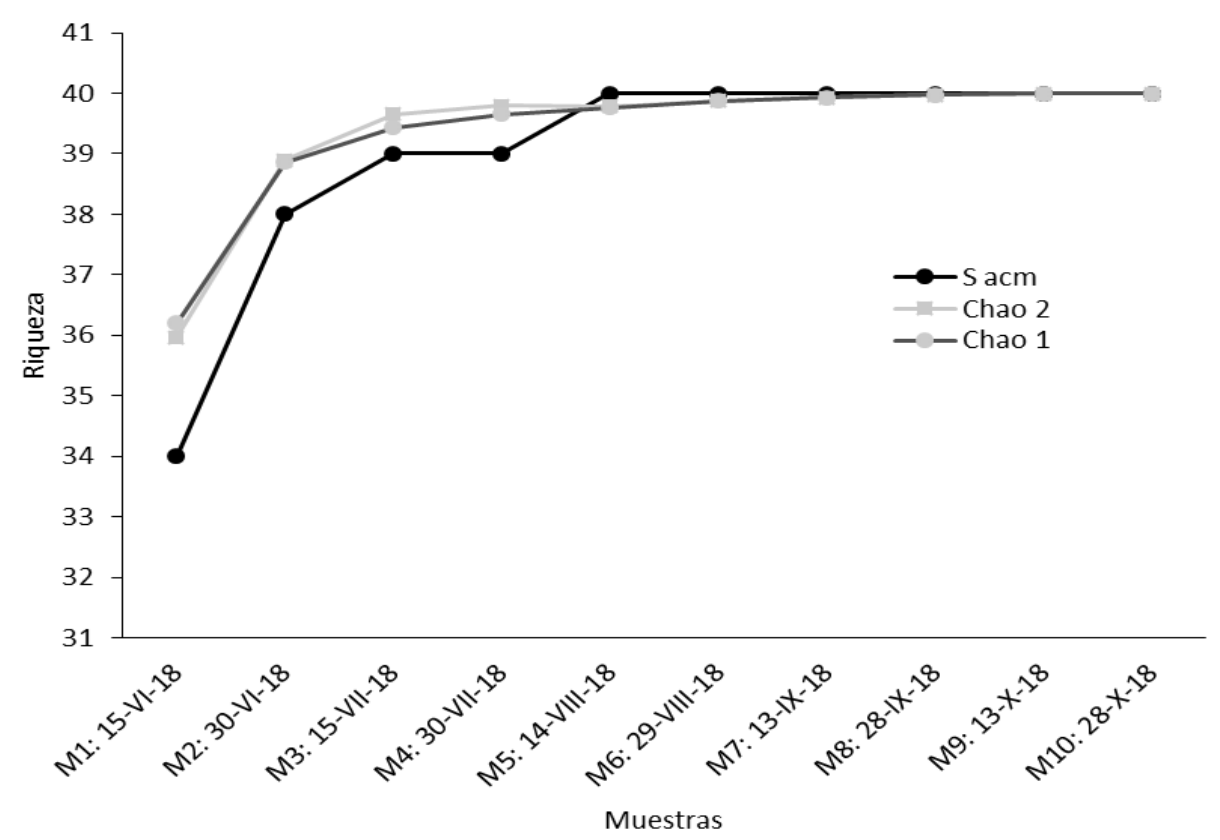

Fig. 3. Curva de acumulación de géneros observados de insectos recolectados en el cultivo de $C$. arabica.

\section{DISCUSIÓN}

La entomofauna registrada en el agroecosistema de café bajo sombra mostró que los coleópteros fueron los insectos más abundantes y esto puede estar asociado al amplio rango alimenticio que ofrecen los cultivos orgánicos, según los reportes realizados en doseles agroforestales, este orden presenta la mayor abundancia (Geraci \& Erwin, 2009). En estudios realizados en plantaciones de café, muestra que es el grupo más abundante y diverso (Perfecto, Vandermeer, Hanson, \& Cartín, 1997; Richter et al., 2007; Aguilar et al., 2019).

La mayoría de los insectos recolectados representan insectos benéficos para el agroecosistema, como las familias Eulophidae e Ichneumonidae (Hymenoptera), que han sido registradas como parasitoides de larvas de lepidópteros, de igual manera los Pentatomidae (Hemiptera), Coccinellidae (Coleoptera), Syrphidae (Diptera) y Chrysopidae (Neuroptera), son depredadores de insectos (Coral, Bacca, \& Dias, 2012).

La alta diversidad de plantas con flores, la disponibilidad de alimento (polen y néctar) y refugio para las abejas podrían estar dando la alta abundancia del género Apis en este agroecosistema (Jha \& Vandermeer, 2009; Zavala et al., 2016). En el caso del género Canthon, los recursos proporcionados, como los frutos caídos (Horgan, 2005; Tribe \& Burger, 2011; Sánchez, Gómez, Delgado, Rodríguez, \& Chamé, 2018), estiércol proporcionado por roedores y otros mamíferos pequeños que usan como refugio y proveen de recursos alimenticios a estos escarabajos (Mendoza \& Horváth, 2013). Además, la falta de competencia en el estrato bajo y el manejo orgánico mantiene la presencia de los insectos y los procesos ecológicos naturales (Cadena, 1991).

Las diferencias registradas en diversidad en los estratos, esta mediada por la presencia de los recursos alimenticios, en nuestro estudio se recolectaron géneros más comunes en el EM y éstos poseen un carácter polífago, capaces de desarrollarse y buscar alimento en el EM y el EA, contrastando con los recolectados en el EB. No existen estudios que aborde la recolecta por estratos en cafetales en la región, se sabe que la mayor diversidad de insectos en los ecosistemas tropicales se registra cerca o en el dosel y la menor abundancia en el estrato bajo (Boiteau, Bousquet, \& 
Osborn, 1999; Basset, Hammond, Barrios, Holloway, \& Miller, 2003; Ulyshen, 2011; McCaig, Sam, Nakamura, \& Stork, 2020).

Las variables ambientales como la temperatura, humedad y precipitación, influyen directa o de manera indirecta en la fluctuación, distribución y establecimiento de los insectos (Wolda \& Fisk, 1981; Dajoz, 2001). Estos datos muestran que la abundancia aumenta cuando se presenta mayor precipitación, elevada humedad y menor temperatura. Se conoce que las precipitaciones altas promueven la producción de hojas tiernas y suculentas en las plantas de las que se alimentan los insectos tropicales (Wolda, 1977; 1978).

La curva de acumulación de especies llego a estabilizarse, lo que indica que se obtuvo mayor registro de insectos, sin embargo, se sugiere incrementar el tiempo de muestreo o usar otros métodos de recolecta para lograr mejor representación de la diversidad de los insectos asociados al café bajo sombra, que es un agroecosistema importante para la conservación de la diversidad, ya que brinda condiciones favorables para la supervivencia de los insectos y otros animales. Este es el primer estudio de la entomofauna asociada a los agroecosistemas de café en la región.

\section{AGRADECIMIENTOS}

Se agradece al Instituto de Investigación para el Desarrollo Sustentable de Ceja de Selva (INDES) de la Universidad Nacional Toribio Rodríguez de Mendoza de Amazonas (UNTRM-A) por el proyecto SNIP No. 209950-PROFITEN con el cual fue posible esta investigación. A Gelber Silva y a Tito Sánchez por la asistencia técnica en este estudio, de igual manera al señor Neyser por el acceso a su agroecosistema. Se agradece a la Estación meteorológica Huambo del INDES-CES de la UNTRMA por la facilidad de los datos climáticos. Agradecemos a los revisores anónimos por sus valiosos comentarios y sugerencias para mejorar la calidad de este artículo.

\section{ÉTICA, CONFLICTO DE INTERESES Y DECLARACIÓN DE FINANCIAMIENTO}

Los autores declaran haber cumplido con los requisitos éticos y legales pertinentes, durante el estudio como en el manuscrito; declaran que no hay conflictos de interés de ningún tipo, y que todas las fuentes financieras se detallan plena y de manera clara en la sección de agradecimientos. Asimismo, están de acuerdo con la versión final del documento. El respectivo documento legal firmado se encuentra en los archivos de la revista.

La contribución de los autores es como se detalla a continuación: A.F.H.P., S.T.L.E. y S.M.O.C.: Diseñaron la investigación, recolectaron, procesaron las muestras e identificaron en material biológico. M.A.H.M.: Diseño la investigación, hizo las corroboraciones de algunos géneros de Coleoptera, realizó los análisis de datos e interpretaciones. Todos los autores contribuyeron durante el proceso de la elaboración y revisión del manuscrito. 


\section{REFERENCIAS}

Aguilar, A. E., Morales, M. C. J., Rosales, E. M. de los A., Gómez, C. J. C., Cena, V. J. M., \& Alonso, B. R. A. (2019). Insectos asociados a cultivos de café en Simojovel de Allende, Chiapas, México. Entomología Mexicana, 6, 379-384.

Anta, F. S. (2006). El café de sombra: un ejemplo de pago de servicios ambientales para proteger la biodiversidad. Gaceta Ecológica, 80, 19-31.

Arnett, Jr. R. H., \& Thomas, M. C. (2000). American beetles. Archostemata, Myxophaga, Adephaga, Polyphaga: Staphyliniformia. Vol. 1. United States of America: CRC Press LLC.

Arnett, Jr. R. H., Thomas, M. C., Skelley, P. E., \& Howard, J. F. (2002). American beetles. Polyphaga: Scarabaeoidea through Curculionoidea. Vol. 2. United States of America: CRC Press LLC.

Barrera, J. F. (2008). Coffee Pests and their Management. In: Capinera J. L. (Ed.), Encyclopedia of Entomology. (pp. 961998). Dordrecht, Netherlands: Springer. DOI: 10.1007/978-1-4020-6359-6

Basset, Y., Hammond, P. M., Barrios, H., Holloway, J. D., \& Miller, S. E. (2003). Vertical stratification of arthropod assemblages. In: Basset, Y. (Ed.), Arthropods of tropical forests: spatio-temporal dynamics and resource use in the canopy. (pp. 17-27). United Kingdom: Cambridge University Press. Cambridge.

Boiteau, G., Bousquet, Y., \& Osborn, W. P. L. (1999). Vertical and temporal distribution of Coccinellidae (Coleoptera) in flight over an agricultural landscape. Canadian Entomologist, 131(2), 269-277. DOI: 10.4039/Ent131269-2

Cadena, G. G. (1991). Sostenibilidad de la producción cafetera, El control biológico de plagas y enfermedades. Ensayos sobre economía cafetera. Federación Nacional de Cafeteros de Colombia, 6(4), 19-31.

Camacho, J., \& Quirós, M. (1995). Clave ilustrada para identificar insectos adultos de las familias del orden Neuroptera (sensu lato) presentes en el occidente de Venezuela. Revista de la Facultad de Agronomía, 12(4), 491-500.

Cheli, G. (1999). Entomología general. Clave para identificar los principales ordenes de la clase Insecto. Recuperado de: https://entomologia.net/L_General.htm

Colwell, R. K. (2013). EstimateS: Statistical estimation of species richness and shared species from samples. Version 9. User's Guide and application. Recuperado de http://purl.oclc.org/estimates

Coral, G. F., Bacca, T., \& Dias, L. G. (2012). Efecto de atractivo de los volátiles de un terpenoide a insectos asociados a Coffea arabica L. (Rubiaceae). Boletín Científico Centro de Museos: Museo de Historia Natural, 16(2), 78-86.

Dajoz, R. (2001). Entomología Forestal: los insectos y el bosque. Madrid, España: Mundi-Prensa.

Fernández, F. (2003). Introducción a las hormigas de la región Neotropical. Bogotá, Colombia: Instituto de Investigación de Recursos Biológicos Alexander von Humboldt.

Foottit, R. G., \& Adler, P. H. (2009). Insect Biodiversity: Science and society. West Sussex, UK: Chennai: Wiley-Blackwell Publishing Ltd.

Geraci, C. J., \& Erwin, T. L. (2009). Amazonian rainforest and their richness of Coleoptera, a dominant life form in the critical zone of the neotropics. In: Adler, P. H. \& Foottit, R. G. (Eds.), Insect Biodiversity. (pp. 49-67). West Sussex, UK: Chennai. Wiley-Blackwell Publishing Ltd.

Horgan, F. G. (2005). Aggregated distribution of resources creates competition refuges for rainforest dung beetle. Ecography, 28(5), 603-618. DOI: 10.1111/j.2005.0906-7590.04177.x

Hortal, J., Borges, P. A. V., \& Gaspar, C. (2006). Evaluating the performance of species richness estimators: Sensitivity to sample grain size. Journal of Animal Ecology, 75(1), 274-287. DOI: 10.1111/j.1365-2656.2006.01048.x 
Jha, S., \& Vandermeer, J. (2009). Contrasting bee foraging in response to resource scale and local habitat management. Oikos, 118(8), 1174-1180. DOI: 10.1111/j.1600-0706.2009.17523.x

Jost, L. (2006). Entropy and diversity. Oikos, 113(2), 363-375. DOI: 10.1111/j.2006.0030-1299.14714.x

Jost, L. (2007). Partitioning diversity into independent alpha and beta components. Ecology, 88(10), 2427-2439. DOI: 10.1890/06-1736.1

Márquez, L. J. (2005). Técnicas de colecta y preservación de insectos. Boletín de la Sociedad Entomológica Aragonesa, 47, 385-408.

McCaig, T., Sam, L., Nakamura, A., \& Stork, N. E. (2020). Is insect vertical distribution in rainforests better explained by distance from the canopy top or distance from the ground?. Biodiversity and Conservation, 29(3), 1081-1103. DOI: 10.1007/s10531-019-01927-0

McGavin, J. C. (2000). Manual de identificación de insectos, arañas y otros artrópodos terrestres. Barcelona, España: Ediciones Omega S. A.

Mendoza, S. V. H., \& Horváth, A. (2013). Roedores y murciélagos en la zona cafetalera del Volcán Tacaná, Chiapas, México. Therya, 4(2), 409-423. DOI: 10.12933/therya-13-116

Mera, V. Y. A., Gallego, R. Ma. C., \& Armbrecht, I. (2010). Interacciones entre hormigas e insectos en follaje de cafetales de sol y sombra, Cauca-Colombia. Revista Colombiana de Entomología, 36(1), 116-126.

Moguel, P., \& Toledo, V. M. (2004). Conservar produciendo: biodiversidad, café orgánico y jardines productivos. Biodiversitas, 55, 2-7.

Moreno, C. E. (2001). Métodos para medir la biodiversidad. M\&T-Manuales y Tesis, Vol. 1. Zaragoza, España: Sociedad Entomológica Aragonesa.

Moreno, C. E., Barragán, F., Pineda, E., \& Pavón, N. P. (2011). Reanálisis de la diversidad alfa: alternativas para interpretar y comparar información sobre comunidades ecológicas. Revista Mexicana de Biodiversidad, 82(4), 1249-1261. DOI: 10.22201 / ib.20078706e.2011.4.745

$\varnothing$ yvind, H., Harper, D. A. T., \& Ryan, P. D. (2001). Past: Paleontological Statistics Software Package for Education and Data Analysis. Palaeontologia Electronica, 4(1), 4-9.

Pérez, De la C. M., Hernández-May, M. A., De la Cruz, P. A., \& Sánchez, S. S. (2016). Scolytinae y Platypodinae (Coleoptera: Curculionidae) de dos áreas de conservación en Tabasco, México. Revista de Biología Tropical, 64(1), 335-342. DOI: 10.15517/rbt.v64i1.15931

Perfecto, I., Rice, R. A., Greenberg, R. \& Van der Voort, M. E. (1996). Shade coffee: A disappearing refuge for biodiversity. BioScience, 46(8), 598-608. DOI: 10.2307/1312989

Perfecto, I., Vandermeer, J., Hanson, P., \& Cartín, V. (1997). Arthropod biodiversity loss and the transformation of a tropical agro-ecosystem. Biodiversity and Conservation, 6, 935-945. DOI: 10.1023/A:1018359429106

Pimentel, D., Stachow, U., Takacs, D. A., Brubaker, H. W., Dumas, A. R., Meaney, J. J., O`neil, J. A. S., Onsi, D. E., \& Corzilius, D. B. (1992). Conserving biological diversity in agricultural/ forestry systems: Most biological diversity exists in human-managed ecosystems. BioScience, 42(5), 354-362. DOI: 10.2307/1311782

Rengífo-Correa, L. A., \& Gonzáles, R. (2011). Clave ilustrada para la identificación de las familias de Pentatomomorpha (Hemiptera: Heteroptera) distribución Neotropical. Boletín Científico Centro de Museos: Museo de Historia natural, 15(1), 168-187.

Resh, V. H., \& Cardé, R. T. (2009). Encyclopedia of Insects. London: Academic press of Elsevier. 
Richter, A., Klein, A. M., Tscharntke, T., \& Tylianakis, J. M. (2007). Abandonement of coffee agroforests increases insect abundance and diversity. Agroforestry Systems, 69(3), 175-182. DOI: 10.1007/s10457-006-9020-y

Ribeyre, F., \& Avelino, J. (2012). Impact of field pests and diseases on coffee quality. In: Oberthür, T., Läderach, P., Jürgen, P. H. A. \& Cock, J. H. (Eds.), Specialty coffee: managing quality. (pp. 151-176). Southeast Asia: Penang: International Plant Nutrition Institute.

Rojas-Briceño, N. B., Barboza, C. E., Maicelo, Q. J. L., Oliva, C. S. M., \& Salas, L. R. (2019). Desforestación en la Amazonía peruana: Índices de cambios de cobertura y uso de suelo basado en SIG. Boletín de la Asociación de Geógrafos Españoles, 81, 2538, 1-34. DOI: 10.21138/bage.2538a

Ruelas, M. L. C., Nava, T. M. E., Cervantes, J., \& Barradas, V. L. (2014). Importancia ambiental de los agroecosistemas cafetaleros bajo sombra en la zona central montañosa del estado de Veracruz, México. Madera y Bosques, 20(3), 27-40.

Sánchez, M. L. (2018). Principales plagas del café y su control. Diplomado en producción sostenible y empresarial de café. Componente de República Dominicana del Programa Centroamericano para la Gestión Integrada del Café (PROCAGICA-RD), 24 pp.

Sánchez, H. G., Gómez, B., Delgado, L., Rodríguez, L. M. E., \& Chamé, V. E. R. (2018). Diversidad de escarabajos copronecrófagos (Coleoptera: Scarabaeidae: Scarabaeinae) en la Reserva de la Biosfera Selva El Ocote, Chiapas, México. Caldasia, 4O(1), 144-160. DOI: 10.15446/caldasia.v40n1.68602

Sanderson, B. A., Svensson, O., Van den Brink, P. J., Gunnarsson, J., \& Tedengren, M. (2018). Insect community composition and functional roles along a tropical agricultural production gradient. Environmental Science and Pollution Research, 25(14), 13426-13438. DOI: 10.1007/s11356-018-1818-4

Tribe, G. D., \& Burger, B. V. (2011). Olfactory ecology. In: Simmons, L. W. \& Ridsdill-Smith T. J. (Eds.), Ecology and evolution of dung beetles. (pp. 87-106). West Sussex, UK: Oxford: Blackwell. DOI: 10.1002/9781444342000.ch5

Triplehorn, C. A., \& Johnson, N. F. (2005). Borror and DeLong's Introduction to the study of insect. United States of America: Thomson Brooks/Cole Cengale learning.

Trjapitzin, V. A., Myartseva, S. N., Ruíz, C. E., \& Coronado, B. J. M. (2008). Clave de géneros de Encyrtidae (Hymenotera: Chalcidoidea) de México y un catálogo de las especies. Ciudad Victoria, Tamaulipas, México: Editorial Planea.

Tuomisto, H. (2010). A consistent terminology for quantifying species diversity? Yes, it does exist. Oecologia, 164(4), 853860. DOI: $10.1007 / \mathrm{s} 00442-010-1812-0$

Tuomisto, H. (2011). Commentary: do we have a consistent terminology for species diversity? Yes, if we choose to use it. Oecologia, 167, 903-911. DOI: 10.1007/s00442-011-2128-4

Ulyshen, M. D. (2011). Arthropod vertical stratification in temperate deciduous forests: implications for conservationoriented management. Forest Ecology and Management, 261, 1479-1489. DOI: 10.1016/j.foreco.2011.01.033

Valarezo, C. O., Cañarte, B. E., \& Navarrete, B. (2012). Artrópodos asociados al cultivo de caco en Manabí. La técnica, 7, $34-42$.

Waller, J. M., Bigger, M., \& Hillocks, R. (2007). Coffee pests, diseases and their management. Coffee pests, diseases and their management. Egham, Surrey, UK: CAB International.

Willie, J., Petre, C. A., Tagg, N., \& Lens, L. (2012). Evaluation of species richness estimators based on quantitative performance measures and sensitivity to patchiness and simple grain size. Acta Oecologica, 45, 31-41. DOI: 10.1016/j.actao.2012.08.004

Wolda, H. (1977). Fluctuations in abundance of some Homoptera in a Neotropical forest. Revue Internationale de Géologie, de Géographie et D'écologie Tropicales, 3, 229-257. 
Wolda, H. (1978). Seasonal fluctuations in rainfall, food and abundance of tropical insects. Journal of Animal Ecology, $47(2), 369-381$.

Wolda, H., \& Fisk, F. W. (1981). Seasonality of tropical insects. II. Blattaria in Panama. Journal of Animal Ecology, 50(3), 827-838.

Young, A. M. (1982). The faunistic richness of insects in the Tropics. In: Young, A. M (Ed.), Population biology of tropical insects. (pp 1-17). New York, E.U.A: Springer, Plenum Press.

Zavala, O. A., Vandame, R., Piana, L., Morales, H., Colomo, G. I., Valle, M. J., \& Villanueva, G. R. (2016). Honey bee (Apis mellifera) foraging ecology in coffee landscapes and description of "coffee garden honey". Journal of Apicultural Research, 55(3), 1-10. DOI: 10.1080/00218839.2016.1215128 